\title{
Mechanical properties of zirconium-based random alloys: alloying elements and composition dependencies
}

\author{
Sunchao Huang, ${ }^{a, b}$ Yongsheng Zhang, ${ }^{a c}$ Xiaoli Zhang, ${ }^{a}$ Zongguo Wang, ${ }^{d}$ Xiaoyu Yang ${ }^{d}$ and Zhi Zeng*ac \\ ${ }^{a}$ Key Laboratory of Materials Physics, Institute of Solid State Physics, Chinese Academy of Sciences, Hefei 230031, China \\ ${ }^{b}$ School of Physics and Material Science, Anhui University, Hefei 230601, China \\ c University of Science and Technology of China, Hefei 230026, China \\ ${ }^{d}$ Computer Network Information Center(CNIC), Chinese Academy of Science Beijing 100190, P.O. Box 349, China
}

\begin{abstract}
We present a detailed investigation on mechanical properties of $\mathrm{Zr}-X$ ( $X=\mathrm{Ti}$, Hf and $\mathrm{Sc}$ ) alloy systems using the first-principles calculations in conjunction with special quasi-random structures (SQSs). It is found that the strength of mechanical coefficients such as elastic constants and elastic modulus depend linearly on the composition in Zr-Hf and Zr-Sc systems, whereas they depend parabolically on the composition in $\mathrm{Zr}-\mathrm{Ti}$ system. Such a phenomenon is mainly induced by the presence of $\mathrm{Zr}-X$ metallic bond in the alloy systems. In addition, the strength of mechanical coefficients can be well described as a function of alloying composition by a special quadratic function that only has one coefficient $F: y=M_{Z r}(1-x)+M_{X} x+x(1-x) F$. With this function, mechanical coefficients of $\mathrm{Zr}-X$ alloy systems in whole composition range can be quickly estimated. Moreover Ti can considerably enhance the ductility of $\mathrm{Zr}$ when $\mathrm{Ti}$ concentration is 0.43 .

Keywords: Zirconium, Alloys, Mechanical properties, Elastic constants, SQSs, First-principles calculations
\end{abstract}

\section{Introduction}

It is known that zirconium ( $\mathrm{Zr})$ and its alloys, possessing low thermal neutron absorption cross section and long-term dimensional stability under irradiation, have attracted great research interests[1, 2, 3]. For any structural material, the strength of mechanical coefficients are crucial parameters for their design and characterization. Experimental ways to obtain these mechanical coefficients are both lengthy and costly in some cases. Alternative ways are theoretical approaches such as the first-principles methods that are widely applied in ordered systems. However, the mechanical properties of the random system were rarely studied by the first-principles methods due to technical difficulties such as how to generate ab initio suitable structure models. The situation had a remarkable turn in 1990, when Zunger and his co-workers proposed a tractable approach on the basis of special quasi-random structures (SQSs) technique to describe random alloys by constructing a finite supercell to match as close as possible with the correlation functions of random alloy[4]. Compared with other commonly employed descriptions, such as the supercells, the cluster expansion (CE)

${ }^{1 *}$ Corresponding author Z.Zeng, E-mail: zzeng@theory.issp.ac.cn 
and the coherent potential approximation (CPA) [5], the SQSs can significantly reduce computation time, acquire comparable accuracy and directly consider local environments. Therefore SQSs are widely used to investigate random alloys and have led to successful research outcomes [5, 6, 7, 8, 9, 10, 11, 12, 13.

It is too hard to investigate all composition points of Zr-based alloys either by experiments or by the first-principles methods. Thus establishing the relationship between composition and properties is rather important. In doing so, in the present study we intend investigating Zr-Ti (titanium), Zr-Hf (hafnium) and Zr-Sc (scandium) random alloy systems by using the first-principles methods in combination with special quasi-random structures. Choosing those alloying systems for this study is based on following facts. Firstly, being the neighbors of $\mathrm{Zr}$ in the periodic table of elements, the transition metals $\mathrm{Ti}$, $\mathrm{Hf}$ and Sc have very similar chemical properties to $\mathrm{Zr}$ and all of them crystallize in hexagonal closed-packed $(h c p)$ structure $(\alpha$ phase) at room temperature. Furthermore, Zr-Ti, Zr-Hf and Zr-Sc alloy systems are characterized by a full solubility of their constituents over a wide temperature range[14. Hence, they are ideal systems to uncover the relationship. For each $\mathrm{Zr}-X$ system, we consider seven structures such as $\mathrm{Zr}_{2}, \mathrm{Zr}_{15} X, \mathrm{Zr}_{12} X_{4}, \mathrm{Zr}_{8} X_{8}$, $\mathrm{Zr}_{4} X_{12}, \operatorname{Zr} X_{15}$ and $X_{2}$. One of theoretical findings from this study is that the mechanical properties of $\mathrm{Zr}-X$ alloys are mainly determined by two factors, namely the alloying constituents and alloying effects. Moreover a mechanical coefficient can be well described via a functional form of alloying composition, given by a special quadratic function: $y=M_{Z r}(1-x)+M_{X} x+x(1-x) F$. By employing this function, the ductility of $\mathrm{Zr}$ can be deduced and can be significantly enhanced through alloying with Ti. In such a case, the enhancement can reach up to a global maximum for Ti concentration at 0.43 .

\section{Theoretical Methods}

\subsection{Computational Details}

Within the framework of density functional theory, the first-principles calculations code of VASP [15] (Vienna Ab initio Simulation Package) is employed to conduct all calculations in the present study. The exchange and correlation potentials are depicted by generalized gradient approximation (GGA) [16] of PerdewBurke-Ernzerhof (PBE). The energy cutoff and k-mesh are chosen to balance accuracy against computational time. Here we use $300 \mathrm{eV}$ as cutoff energy for all calculations, $(13 \times 13 \times 8) \mathrm{k}$-mesh for structures $\mathrm{Zr}_{15} X, \mathrm{Zr}_{8} X_{8}$, $\operatorname{Zr} X_{15}$, and $(25 \times 25 \times 17) \mathrm{k}$-mesh for $\mathrm{Zr}_{2}$ as well as $X_{2}$, and $(11 \times 13 \times 6)$ for structures $\mathrm{Zr}_{12} X_{4}$ and $\mathrm{Zr}_{4} X_{12}$. Elastic constants are calculated by stress-strain approach, i.e., applying a number of strains and calculating corresponding stress, then obtaining elastic constants by solving generalized Hooke law. More details about this approach have been documented in the literature[17].

\subsection{The $S Q S$ Template}

The technique of SQSs was proposed by Zunger and his co-workers 4. One of its major advantages is that this approach can considerably overcome the computational difficulties in modeling random alloys by constructing a finite supercell to mimic as close as possible to the correlation functions of a random alloy. The 

of element $B, k$ is the number of vertices and $m$ is the maximum distance spanned by the figure edge in units of nearest-neighbor shells. For $h c p$ structure with an ideal c/a ratio, although the atom-pair $(0,0,0)$ and $(1,0,0)$ has the same interatomic distance with the atom-pair $(0,0,0)$ and $(1 / 3,2 / 3,1 / 2)$, they have different symmetry operations. In addition, the c/a ratio is not ideal for majority of hexagonal structures, leading to differences between these two pairs. Thus, the index $n$ is employed to distinguish them. The correlation functions of SQSs are defined with the Ising model language and the details about this can be found in literatures [4, 5, 18, 19]. The quality of SQSs depends on how their correlation functions come up with the correlation functions of the random alloy.

\subsection{Projection of Elastic Tensor to Those of Higher Symmetry}

It is well known that the symmetry of an elastic tensor is controlled by atomic arrangements in microstructure. For example, [100] and [010] directions of $h c p$ structures possess the same atomic arrangements, which leads to $C_{11}=C_{22}$. For $h c p$ type of random alloys, [100] and [010] are not the exactly equivalent directions owing to random occupation of atoms in the lattice sites. However they still have the same average environments and are supposed to hold $C_{11}=C_{22}$, which means that the elastic tensors of random alloys own the same symmetry as those of ordered phase. Normally, the size of SQSs used to mimic random alloys is finite that leads to symmetrical directions having different average environments and introducing a part of symmetry deviations. In addition, the first-principles approach will also introduce some symmetry deviations. As a consequence, calculated elastic tensor having 21 independent components differs from the experimental one that only has 5 independent components. Rather than ignoring the extra 16 independent components, here we would like to introduce a projection technique that was proposed by Moakher et al. 20] to project calculated elastic tensor in order to have the same form as the experimental one. The key principle of this technique is using a series of point group operations in obtaining a higher symmetry elastic tensor from one with arbitrary symmetry. Technically, those point group operations are presented by projector $P^{s y m}$. Here $h c p$ type of projector $P^{h c p}$ is employed,

$$
P^{h c p}=\left(\begin{array}{cc}
Q^{h c p} & 0_{9 \times 12} \\
0_{12 \times 9} & 0_{12 \times 12}
\end{array}\right),
$$


where $Q^{h c p}$ is

$$
\left(\begin{array}{ccccccccc}
3 / 8 & 3 / 8 & 0 & 0 & 0 & 1 / 4 \sqrt{2} & 0 & 0 & 1 / 4 \\
3 / 8 & 3 / 8 & 0 & 0 & 0 & 1 / 4 \sqrt{2} & 0 & 0 & 1 / 4 \\
0 & 0 & 1 & 0 & 0 & 0 & 0 & 0 & 0 \\
0 & 0 & 0 & 1 / 2 & 1 / 2 & 0 & 0 & 0 & 0 \\
0 & 0 & 0 & 1 / 2 & 1 / 2 & 0 & 0 & 0 & 0 \\
1 / 4 \sqrt{2} & 1 / 4 \sqrt{2} & 0 & 0 & 0 & 3 / 4 & 0 & 0 & -1 / 2 \sqrt{2} \\
0 & 0 & 0 & 0 & 0 & 0 & 1 / 2 & 1 / 2 & 0 \\
0 & 0 & 0 & 0 & 0 & 0 & 1 / 2 & 1 / 2 & 0 \\
1 / 4 & 1 / 4 & 0 & 0 & 0 & -1 / 2 \sqrt{2} & 0 & 0 & 1 / 2
\end{array}\right) .
$$

In order to carry out the projection, firstly, the elastic tensor should be written as a vector with 21 components, which reads

$$
\begin{gathered}
C=\left(C_{11}, C_{22}, C_{33}, \sqrt{2} C_{23}, \sqrt{2} C_{13}, \sqrt{2} C_{12}, 2 C_{44},\right. \\
2 C_{55}, 2 C_{66}, 2 C_{14}, 2 C_{25}, 2 C_{36}, 2 C_{34}, 2 C_{15}, 2 C_{26}, \\
\left.2 C_{24}, 2 C_{35}, 2 C_{16}, 2 \sqrt{2} C_{56}, 2 \sqrt{2} C_{46}, 2 \sqrt{2} C_{45}\right), \\
C^{h c p}=P^{h c p} C .
\end{gathered}
$$

Here the numerical coefficients $(2$ and $\sqrt{2})$ are employed to ensure the invariance of the Euclidean norm[20]. Using equation (3), the projected elastic constants tensor $C^{\text {hcp }}$ can be obtained as following,

$$
\begin{aligned}
C^{h c p}= & \left(\bar{C}_{11}, \bar{C}_{11}, \bar{C}_{33}, \sqrt{2} \bar{C}_{13}, \sqrt{2} \bar{C}_{13}, \sqrt{2} \bar{C}_{12}, 2 \bar{C}_{44},\right. \\
& \left.2 \bar{C}_{44}, \bar{C}_{11}-\bar{C}_{12}, 0,0,0,0,0,0,0,0,0,0,0,0\right),
\end{aligned}
$$

where projected elastic constants $\bar{C}_{11}, \bar{C}_{33}, \bar{C}_{44}, \bar{C}_{12}, \bar{C}_{13}$ are determined by following equations

$$
\begin{array}{r}
\bar{C}_{11}=3\left(C_{11}+C_{22}\right) / 8+C 12 / 4+C_{66} / 2, \bar{C}_{33}=C_{33}, \bar{C}_{44}=\left(C_{44}+C_{55}\right) / 2 \\
\bar{C}_{12}=\left(C_{12}+C_{22}\right) / 8+3 C_{12} / 4-C_{66} / 2, \bar{C}_{13}=\left(C_{13}+C_{23}\right) / 2 .
\end{array}
$$

The Euclidean distance deviations $P$ [11] can be employed to characterize the degree of symmetry deviations, which is defined as

$$
P=\left\|C-C^{h c p}\right\| /\|C\| .
$$

For convenience, from now on all projected elastic constants $\bar{C}_{i j}$ will be denoted as $C_{i j}$ and the unprojected elastic constants will not be mentioned any more.

\section{Results and Discuss}

The SQS approach has been widely employed to investigate thermodynamic properties of material systems such as [5, 7, 19, 21, 22]. There are already some SQSs templates with arbitrary supercell shape $(b c c[5], f c c[22]$ 
and $h c p[18]$ ) in the literatures. However, when SQS approach was employed to investigate elastic constants previously, SQSs were usually reconstructed[10, 12] in order to possess $A \times A \times A$ supercell shape because elastic constants are sensitive to the symmetry of the model[11]. In fact, SQSs employed to investigate elastic constants firstly should own acceptable correlation functions, and then should own $A \times A \times A$ supercell shape that is suggested but not obligatory. Based on this rule, two types of SQSs-16 (Fig. 1 and Table I) are generated for $\mathrm{Zr}-X$ alloys with the alloy theoretic automation toolkit (ATAT). The first one keeps a $2 \times 2 \times 2$ supercell, built for $\mathrm{Zr}_{8} X_{8}$, which balances the symmetry of the model to against the correlation functions. The another one, built for $\mathrm{Zr}_{4} X_{12}$ and $\mathrm{Zr}_{12} X_{4}$, does not keep a $2 \times 2 \times 2$ supercell in order to acquire the acceptable correlation functions. Its lattice vectors $(a)$ can be linked with those of $h c p$ unit cell $(c)$ via an equation $a=b c$ where matrix $b$ is given as

$$
\left(\begin{array}{ccc}
1 & 0 & -1 \\
1 & -2 & 0 \\
-3 & 0 & -1
\end{array}\right) .
$$

As a result, our SQSs applied in this study have following characteristics: (a) the comparable correlation functions among different concentrations, (b) $2 \times 2 \times 2$ supercell shape at concentration $x=0.5$. Fig. $1(a)$ is the schematic diagram of $\mathrm{Zr}_{12} X_{4}(x=0.25)$ and $\mathrm{Zr}_{4} X_{12}(x=0.75)$, and Fig. $1(b)$ is the schematic diagram of $\mathrm{Zr}_{8} X_{8}(x=0.5)$ where an orange plane and four blue planes are employed to divide it into 8 hcp unit cells. The lattice vectors and atomic sites of SQSs in fractional coordinates are given in Table I. Although the SQS at a concentration $x=0.5$ possesses $2 \times 2 \times 2$ supercell shape, the correlation functions agree well with those reported by Shin et al. [18, (TableII). For our SQSs, the average two-body correlation functions of $\mathrm{Zr}_{8} X_{8}$ equal to those of a random alloy from the first- to the fourth-nearest-neighbor, and the average three-body as well as the average four-body correlation functions equal to those of the third- and the secondnearest-neighbor respectively. The average two-body correlation functions of $\mathrm{Zr}_{12} X_{4}\left(\mathrm{Zr}_{4} X_{12}\right)$ equal to those of random alloy for pairs from the first- to the fourth-nearest-neighbor. These guarantee a high quality of SQS templates to mimic random alloys.

Based on above shown structures, mechanical properties of Zr-Ti, Zr-Hf and Zr-Sc alloy systems, such as, elastic constants, bulk modulus and shear modulus, are systematically investigated. Elastic constants, which can be directly calculated by the first-principles approach, are crucial mechanical parameters which link stress with strain. In addition, a number of properties can be derived from them. In the present work, we firstly calculate elastic tensor. Then by applying projection technique, calculated tensor is projected to satisfy hcp symmetry. Bulk modulus $(B)$ and shear modulus $(G)$ are obtained in terms of Voigt-Reuss-Hill approach 23]. The $B / G$ rate and Poisson's ratio $\nu$ are also calculated to examine the ductility where we should notice that about $1.75[23$ for $B / G$ and 0.3 for $\nu$ are crucial points to separate ductile $(B / G>1.75$ and $\nu>0.3)$ and brittle materials $(B / G<1.75$ and $\nu<0.3)$.

The results for the elastic constants, bulk modulus $(B)$, shear modulus $(G), B / G$ and Poisson's ratio $\nu$ from the present study $v s$ accessible experimental values and other theoretical data for pure metals are listed 
Table 1: Lattice vectors and atomic positions of structures, which are given in their ideal, unrelaxed states

\begin{tabular}{|c|c|c|}
\hline $\mathrm{Zr}_{15} X_{1}\left(\mathrm{Zr}_{1} X_{15}\right)$ & $\mathrm{Zr}_{12} X_{4}\left(\mathrm{Zr}_{4} X_{12}\right)$ & $\mathrm{Zr}_{8} X_{8}$ \\
\hline lattice vectors & lattice vectors & lattice vectors \\
\hline$\left(\begin{array}{lll}6.510 & 0.000 & 0.000\end{array}\right)$ & $\left(\begin{array}{lll}3.255 & 0.000 & -5.315\end{array}\right)$ & $\left(\begin{array}{lll}6.510 & 0.000 & 0.000\end{array}\right)$ \\
\hline$\left(\begin{array}{lll}3.255 & 5.638 & 0.000\end{array}\right)$ & 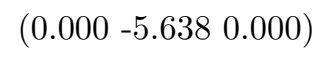 & $\left(\begin{array}{lll}3.255 & 5.638 & 0.000\end{array}\right)$ \\
\hline$\left(\begin{array}{lll}0.000 & 0.000 & 10.631\end{array}\right)$ & $(-9.7650 .000-5.315)$ & $\left(\begin{array}{lll}0.000 & 0.000 & 10.631\end{array}\right)$ \\
\hline Atomic positions & Atomic positions & Atomic positions \\
\hline A-( $\left(\begin{array}{lll}1 & 0 & 0\end{array}\right)$ & A-(1/8 1/2 7/8) & $\mathrm{A}-\left(\begin{array}{lll}1 & 0 & 0\end{array}\right)$ \\
\hline $\mathrm{A}-(1 / 61 / 61 / 4)$ & A- $(7 / 8$ 1/3 5/8) & $\mathrm{A}-(1 / 61 / 61 / 4)$ \\
\hline $\mathrm{A}-\left(\begin{array}{lll}1 / 2 & 0 & 0\end{array}\right)$ & A-(3/8 1/2 5/8) & $\mathrm{A}-\left(\begin{array}{lll}1 / 2 & 0 & 0\end{array}\right)$ \\
\hline $\mathrm{A}-(2 / 3$ 1/6 1/4) & $\mathrm{A}-(3 / 8$ 1/3 1/8) & $\mathrm{A}-(2 / 3 \quad 1 / 61 / 4)$ \\
\hline $\mathrm{A}-\left(\begin{array}{lll}1 / 2 & 1 / 2 & 0\end{array}\right)$ & $\mathrm{A}-(7 / 8$ 1/2 1/8) & $\mathrm{A}-\left(\begin{array}{lll}1 / 2 & 1 / 2 & 0\end{array}\right)$ \\
\hline $\mathrm{A}-(2 / 32 / 3$ 1/4) & A- $(5 / 81 / 37 / 8)$ & $\mathrm{A}-(2 / 3 \quad 2 / 3$ 1/4) \\
\hline $\mathrm{A}-\left(\begin{array}{lll}1 & 0 & 1 / 2\end{array}\right)$ & 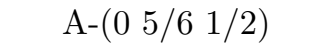 & $\mathrm{A}-\left(\begin{array}{lll}1 & 0 & 1 / 2\end{array}\right)$ \\
\hline $\mathrm{A}-(1 / 61 / 63 / 4)$ & $\mathrm{A}-(1 / 45 / 61 / 4)$ & $\mathrm{A}-(1 / 61 / 63 / 4)$ \\
\hline $\mathrm{A}-(1 / 62 / 33 / 4)$ & $\mathrm{A}-(3 / 401 / 4)$ & B-(1/6 2/3 3/4) \\
\hline$A-\left(\begin{array}{lll}1 & 1 / 2 & 1 / 2\end{array}\right)$ & $\mathrm{A}-(1 / 25 / 60)$ & B-(1 1/2 1/2) \\
\hline $\mathrm{A}-(2 / 32 / 33 / 4)$ & $\mathrm{A}-\left(\begin{array}{lll}0 & 0 & 0\end{array}\right)$ & B- $(2 / 32 / 33 / 4)$ \\
\hline$A-\left(\begin{array}{lll}1 & 1 / 2 & 0\end{array}\right)$ & $\mathrm{A}-(3 / 45 / 63 / 4)$ & B-(1/2 1/2 1/2) \\
\hline $\mathrm{A}-(2 / 31 / 63 / 4)$ & B-( $\left(\begin{array}{lll}1 / 2 & 0 & 1 / 2\end{array}\right)$ & $\mathrm{B}-(2 / 31 / 63 / 4)$ \\
\hline $\mathrm{A}-\left(\begin{array}{lll}1 / / 2 & 0 & 1 / 2\end{array}\right)$ & $\mathrm{B}-(1 / 403 / 4)$ & $\mathrm{B}-(1 / / 2 \quad 01 / 2)$ \\
\hline $\mathrm{A}-(1 / 62 / 31 / 4)$ & B-(5/8 1/2 3/8) & $\mathrm{B}-(1 / 62 / 31 / 4)$ \\
\hline B-(1/2 1/2 1/2) & B-(1/8 1/3 3/8) & B-( $\left(\begin{array}{lll}1 & 1 / 2 & 0\end{array}\right)$ \\
\hline
\end{tabular}


Table 2: Pair and multi-site correlation functions of $\mathrm{Zr}_{8} X_{8}$ and $\mathrm{Zr}_{12} X_{4}$ configurations when the c/a ratio is ideal. The number in the square bracket next to $\bar{\Pi}_{k, m, n}$ is the degeneracy factor at the same distance with crystallographically equivalent.

\begin{tabular}{|c|c|c|c|c|c|c|}
\hline & \multicolumn{3}{|c|}{$\mathrm{Zr}_{8} X_{8}$} & \multicolumn{3}{|c|}{$\mathrm{Zr}_{12} X_{4}\left(\mathrm{Zr}_{4} X_{12}\right)$} \\
\hline & Random & Ref[18] & Present Work & Random & $\operatorname{Ref}[18]$ & Present Work \\
\hline $\bar{\Pi}_{2,1,1}[6]$ & 0.0000 & 0.0000 & 0.0000 & 0.2500 & 0.2500 & 0.2500 \\
\hline $\bar{\Pi}_{2,1,2}[6]$ & 0.0000 & 0.0000 & 0.0000 & 0.2500 & 0.2500 & 0.2500 \\
\hline $\bar{\Pi}_{2,2,1}[6]$ & 0.0000 & 0.0000 & 0.0000 & 0.2500 & 0.2500 & 0.2500 \\
\hline $\bar{\Pi}_{2,3,1}[2]$ & 0.0000 & 0.0000 & 0.0000 & 0.2500 & 0.2500 & 0.2500 \\
\hline $\bar{\Pi}_{2,4,1}[12]$ & 0.0000 & 0.0000 & 0.0000 & 0.2500 & 0.2500 & 0.2500 \\
\hline $\bar{\Pi}_{2,4,2}[6]$ & 0.0000 & 0.0000 & 0.0000 & 0.2500 & 0.4583 & 0.3333 \\
\hline $\bar{\Pi}_{2,5,1}[12]$ & 0.0000 & 0.0000 & -0.3333 & 0.2500 & 0.3333 & 0.4583 \\
\hline $\bar{\Pi}_{2,6,1}[6]$ & 0.0000 & -0.3333 & 1.0000 & 0.2500 & 0.1667 & 0.1667 \\
\hline $\bar{\Pi}_{2,7,1}[12]$ & 0.0000 & 0.0000 & 0.0000 & 0.2500 & 0.2500 & 0.2500 \\
\hline $\bar{\Pi}_{2,8,1}[12]$ & 0.0000 & 0.0000 & -0.3333 & 0.2500 & 0.1667 & 0.1667 \\
\hline $\bar{\Pi}_{3,1,1}[12]$ & 0.0000 & 0.0000 & 0.0000 & 0.1250 & -0.0833 & -0.1667 \\
\hline $\bar{\Pi}_{3,1,2}[2]$ & 0.0000 & 0.0000 & 0.0000 & 0.1250 & 0.2500 & -0.2500 \\
\hline $\bar{\Pi}_{3,1,3}[2]$ & 0.0000 & 0.0000 & 0.0000 & 0.1250 & 0.2500 & -0.2500 \\
\hline $\bar{\Pi}_{3,2,1}[24]$ & 0.0000 & 0.0000 & 0.0000 & 0.1250 & -0.042 & -0.1250 \\
\hline $\bar{\Pi}_{3,3,1}[6]$ & 0.0000 & 0.0000 & 0.0000 & 0.1250 & -0.0833 & -0.0833 \\
\hline $\bar{\Pi}_{4,1,1}[4]$ & 0.0000 & 0.0000 & 0.0000 & 0.0625 & -0.0833 & 0.2500 \\
\hline $\bar{\Pi}_{4,2,1}[12]$ & 0.0000 & 0.0000 & 0.0000 & 0.0625 & -0.1667 & 0.1667 \\
\hline $\bar{\Pi}_{4,2,2}[12]$ & 0.0000 & 0.0000 & 0.0000 & 0.0625 & 0.0000 & 0.0000 \\
\hline $\bar{\Pi}_{4,3,1}[6]$ & 0.0000 & 0.3333 & -0.3333 & 0.0625 & -0.1667 & 0.0833 \\
\hline
\end{tabular}


Table 3: Calculated elastic constants $C_{11}, C_{33}, C_{12}, C_{13}, C_{44}$, bulk modulus $(B)$, shear modulus $(G)$, Poisson's ratio $(\nu)$, accessible experimental and theoretical values are also listed.

\begin{tabular}{|c|c|c|c|c|c|c|c|c|c|c|}
\hline & $\begin{array}{c}C_{11} \\
(G P a)\end{array}$ & $\begin{array}{c}C_{33} \\
(G P a)\end{array}$ & $\begin{array}{c}C_{12} \\
(G P a)\end{array}$ & $\begin{array}{c}C_{13} \\
(G P a)\end{array}$ & $\begin{array}{c}C_{44} \\
(G P a)\end{array}$ & $\begin{array}{c}B \\
(G P a)\end{array}$ & $\begin{array}{c}G \\
(G P a)\end{array}$ & $\nu$ & $\mathrm{B} / \mathrm{G}$ & $\begin{array}{c}P \\
(\%)\end{array}$ \\
\hline $\mathrm{Zr}$ & 144.106 & 163.926 & 70.332 & 71.664 & 23.419 & 97.432 & 31.468 & 0.354 & 3.096 & 1.049 \\
\hline Zr (Theory) 23. & 145.300 & 166.100 & 67.300 & 69.500 & 24.300 & 96.589 & 31.273 & 0.354 & 3.089 & \\
\hline $\operatorname{Zr}(\operatorname{Exp}, 298 K) 24$ & 143.400 & 164.800 & 72.800 & 65.300 & 32.000 & 95.378 & 35.747 & 0.333 & 2.668 & \\
\hline $\mathrm{Zr}_{15} \mathrm{Ti}_{1}$ & 144.946 & 163.108 & 70.628 & 70.588 & 25.470 & 97.270 & 32.926 & 0.348 & 2.954 & 0.064 \\
\hline $\mathrm{Zr}_{12} \mathrm{Ti}_{4}$ & 130.633 & 157.202 & 81.304 & 75.505 & 28.838 & 98.110 & 27.386 & 0.372 & 3.583 & 7.286 \\
\hline $\mathrm{Zr}_{8} \mathrm{Ti}_{8}$ & 135.753 & 163.377 & 86.560 & 78.644 & 25.721 & 101.709 & 26.844 & 0.379 & 3.789 & 4.182 \\
\hline $\mathrm{Zr}_{4} \mathrm{Ti}_{12}$ & 145.073 & 173.469 & 90.130 & 80.529 & 31.739 & 107.322 & 32.209 & 0.364 & 3.332 & 5.523 \\
\hline $\mathrm{Zr}_{1} \mathrm{Ti}_{15}$ & 167.734 & 181.558 & 87.462 & 82.973 & 37.363 & 113.747 & 40.383 & 0.341 & 2.817 & 0.087 \\
\hline $\mathrm{Ti}$ & 179.019 & 189.534 & 87.912 & 82.640 & 40.511 & 116.991 & 44.598 & 0.331 & 2.623 & 0.838 \\
\hline Ti (Theory) 23] & 174.800 & 189.500 & 87.500 & 80.000 & 40.900 & 114.900 & 43.980 & 0.330 & 2.613 & \\
\hline $\mathrm{Ti}(\operatorname{Exp}, 298 K) 24$ & 162.400 & 180.700 & 92.000 & 69.000 & 46.700 & 107.278 & 46.387 & 0.311 & 2.313 & \\
\hline $\mathrm{Zr}_{15} \mathrm{Hf}_{1}$ & 148.904 & 166.451 & 68.958 & 71.423 & 25.124 & 98.483 & 33.849 & 0.346 & 2.910 & 0.073 \\
\hline $\mathrm{Zr}_{12} \mathrm{Hf}_{4}$ & 156.402 & 171.194 & 69.914 & 70.931 & 32.286 & 100.826 & 39.273 & 0.328 & 2.567 & 0.892 \\
\hline $\mathrm{Zr}_{8} \mathrm{Hf}_{8}$ & 165.226 & 179.999 & 70.006 & 72.904 & 37.042 & 104.545 & 43.642 & 0.317 & 2.396 & 0.477 \\
\hline $\mathrm{Zr}_{4} \mathrm{Hf}_{12}$ & 176.332 & 189.753 & 73.217 & 72.031 & 46.640 & 108.486 & 50.446 & 0.299 & 2.151 & 0.870 \\
\hline $\mathrm{Zr}_{1} \mathrm{Hf}_{15}$ & 184.110 & 192.695 & 72.694 & 73.701 & 50.374 & 111.200 & 53.923 & 0.291 & 2.062 & 0.062 \\
\hline $\mathrm{Hf}$ & 185.995 & 196.981 & 73.666 & 73.893 & 52.403 & 112.413 & 55.264 & 0.289 & 2.034 & 0.201 \\
\hline Hf (Theory) 23] & 183.600 & 197.900 & 71.100 & 71.100 & 51.100 & 110.189 & 54.113 & 0.289 & 2.036 & \\
\hline Hf $(\operatorname{Exp}, 298 K)[24$ & 188.100 & 196.900 & 77.200 & 66.100 & 55.700 & 110.211 & 57.667 & 0.277 & 1.911 & \\
\hline $\mathrm{Zr}_{15} \mathrm{Sc}_{1}$ & 153.311 & 160.871 & 58.689 & 66.733 & 31.068 & 94.547 & 39.475 & 0.317 & 2.395 & 0.047 \\
\hline $\mathrm{Zr}_{12} \mathrm{Sc}_{4}$ & 146.638 & 151.275 & 50.690 & 56.694 & 37.006 & 85.914 & 42.591 & 0.287 & 2.017 & 1.019 \\
\hline $\mathrm{Zr}_{8} \mathrm{Sc}_{8}$ & 130.239 & 134.452 & 44.285 & 46.389 & 33.149 & 74.436 & 39.055 & 0.277 & 1.906 & 2.874 \\
\hline $\mathrm{Zr}_{4} \mathrm{Sc}_{12}$ & 115.445 & 115.544 & 39.906 & 36.707 & 32.257 & 63.548 & 35.914 & 0.262 & 1.769 & 1.555 \\
\hline $\mathrm{Zr}_{1} \mathrm{Sc}_{15}$ & 105.388 & 103.926 & 37.238 & 29.760 & 30.341 & 56.438 & 33.359 & 0.253 & 1.692 & 0.179 \\
\hline $\mathrm{Sc}$ & 102.125 & 100.433 & 36.750 & 27.034 & 30.461 & 53.973 & 32.850 & 0.247 & 1.643 & 0.000 \\
\hline Sc (Theory) 23 & 104.800 & 105.200 & 37.700 & 29.300 & 31.600 & 56.378 & 33.527 & 0.252 & 1.682 & \\
\hline
\end{tabular}


Figure 1: (Color online) Schematic diagrams of SQSs-16, where red and green spheres represent $\mathrm{Zr}$ and $X$ atoms, respectively. (a) for $\mathrm{Zr}_{4} X_{12}\left(\mathrm{Zr}_{12} X_{4}\right)$ with $a=5.934 \AA, b=5.535 \AA, c=10.696 \AA, \alpha=89^{\circ}, \beta=94^{\circ}$ and $\gamma=90^{\circ}$ (b) for $\mathrm{Zr}_{8} X_{8}$ with $2 \times 2 \times 2$ supercell shape $\left(a=b=6.510 \AA, c=10.631 \AA, \alpha=\beta=90^{\circ}\right.$ and $\gamma=120^{\circ}$, where an orange plane and four blue planes are employed to divide it into 8 hcp unit cell.

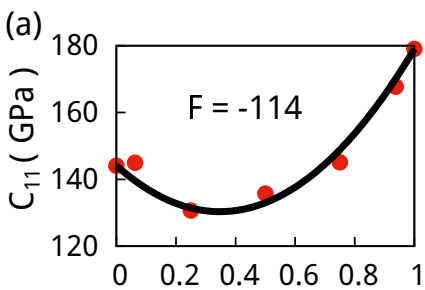

(b)
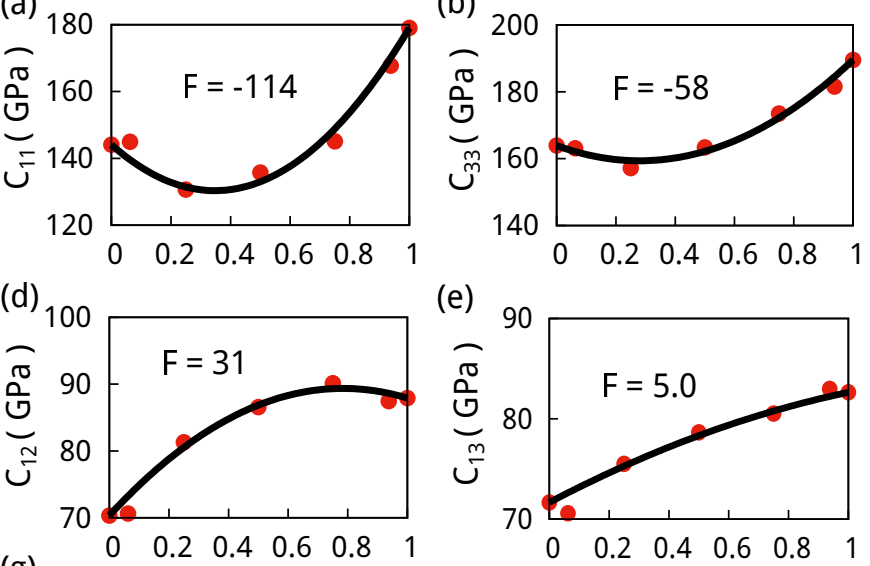

(g)

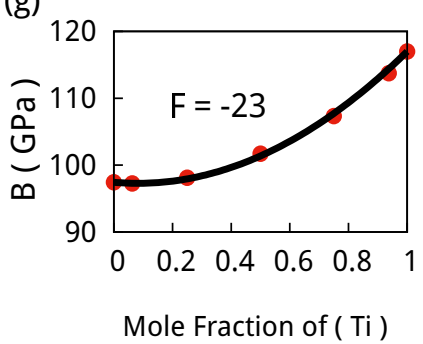

(e)

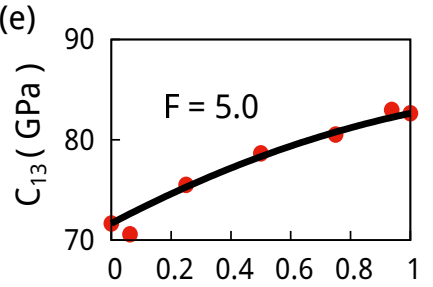

(h)

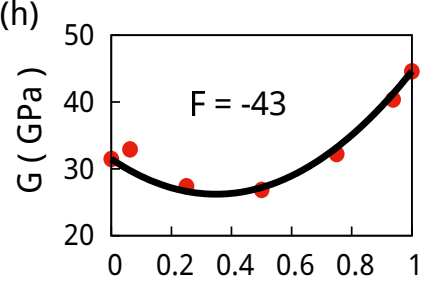

Mole Fraction of ( $\mathrm{Ti}$ )
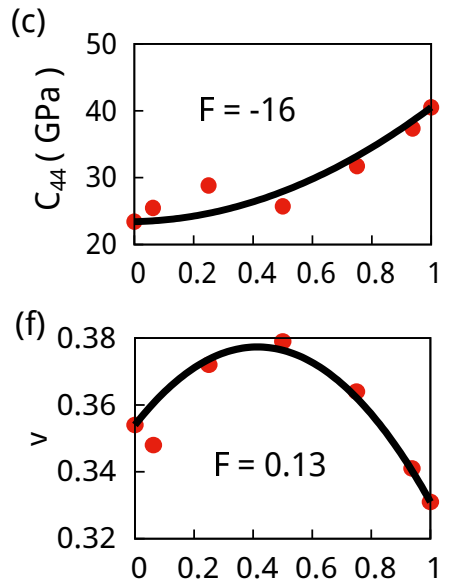

(i)

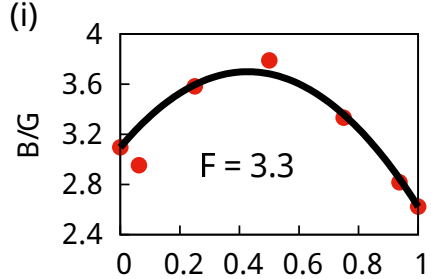

Mole Fraction of ( $\mathrm{Ti}$ )

Figure 2: (Color online) Mechanical properties of Zr-Ti alloy system (a) $C_{11}$, (b) $C_{33}$, (c) $C_{44}$, (d) $C_{12}$, (e) $C_{13}$, (f) $\nu$, (g) $B$, (h) $G$ and (i) $B / G$ The red spots are calculated values and the black lines are fitting function $y=M_{Z_{r}}(1-x)+M_{X} x+x(1-x) F$ with parameter $F$.

in Table III, from where we can see that a good agreement between our results and those in the literature values can be found. For example, calculated $C_{11}(144.106 \mathrm{GPa})$ for $\mathrm{Zr}_{2}$ agrees well with the experimental datum (143.400 GPa). To the best of our knowledge, there are no experimental data for elastic constants in random alloys [10, 11, 12. Although Hsueh-Chuan Hsu etal. has prepared a series of binary Zr-Ti alloys and 
measured some of their mechanical properties such as hardness and bending moduli, elastic constants have not yet been measured experimentally [25]. Meanwhile, those Zr-Ti alloys were neither random nor single crystals. Their results showed that the bending moduli changed with varying Ti concentration. Nevertheless the variation tendency was undistinguishable due to experimental errors. Based on the calculated elastic constants, the mechanical stability can be judged according to Born's criteria. For a hexagonal system,

$$
\begin{aligned}
C_{11}-\left|C_{12}\right| & >0 \\
\left(C_{11}+C_{12}\right) C_{13}-2 C_{12}^{2} & >0 \\
C_{44} & >0
\end{aligned}
$$

It is clear that all of the $\mathrm{Zr}-X$ alloy systems are mechanically stable. The obtained B/G (last but one column in Table III) indicates that $\mathrm{Zr}-\mathrm{Ti}$ and $\mathrm{Zr}-\mathrm{Hf}$ are ductile materials. Although Sc is a brittle material, ScZr alloys are ductile materials when $\mathrm{Zr}$ concentration is great than 0.25 .

To analyze the composition dependence, mechanical properties as a function of alloying concentration are fitted with a quadratic function which just has one parameter $F$,

$$
y=M_{Z r}(1-x)+M_{X} x+x(1-x) F,
$$

where $y$ is the mechanical property of $\operatorname{Zr}_{(1-x)} X_{x}$ alloy, $M_{Z r}$ is the mechanical property of $\mathrm{Zr}, M_{X}$ is the mechanical property of alloying elements $X\left(X=\mathrm{Ti}\right.$, Hf and Sc). The physical meaning of $M_{Z_{r}}(1-x)$ and $M_{X} x$ are the contribution to mechanical properties of $\mathrm{Zr}_{(1-x)} X_{x}$ from pure $\mathrm{Zr}$ and pure $X$ metal respectively. $F$ is the contribution of interaction between a pair of $\mathrm{Zr}$ and $X$ atoms to the mechanical properties of $\operatorname{Zr}_{(1-x)} X_{x}$ and $x(1-x)$ represents the number of pairs of $\mathrm{Zr}$ and $X$. Thus the physical meaning of $x(1-x) F$ is the contribution from all of interactions between $\mathrm{Zr}$ and $X$ to mechanical properties of $\mathrm{Zr}_{1-x} X_{x}$. This function decomposed the strength of mechanical properties into two parts: the first one is determined by alloying constituents and the second one is determined by alloying effect. The results of fitting are shown in Fig. 2 and Fig.3, from where we can see that all mechanical properties change apparently with alloying concentration. Taking $B / G$ rate for instance, it shows nonlinear dependence on alloying composition with a global maximum around $\mathrm{Ti}$ concentration at 0.43 in $\mathrm{Zr}$-Ti alloy system (Fig. 2(i)), while it gradually decreases with increasing alloying elements in Zr-Hf (Fig. 3(a)) and Zr-Sc (Fig. 3(b)) systems.

The performance of Eq. (8) for Zr-Ti system is shown in Fig. 2. For elastic constants, $C_{i i}$ exhibits concave curves, while $C_{i j}(i \neq j)$ exhibits convex curves. $B / G$ and $\nu$ reveal convex curves as well, which means that Zr alloying with Ti can significantly improve the ductility. By taking the first derivative of fitting function, the global maximum of $B / G$, where the enhancement of the ductility is the best, can be found. Here the fitting function for $B / G$ is $y=3.096(1-x)+2.623 x+3.29 x(1-x)$ and the global maximum is at $x=0.43$. The first two terms, contribution from pure constituent, decrease with $x$, while the last term, contribution from alloying effects, firstly increases with $x \in(0,0.5)$, and then decreases with $x \in(0.5,1)$. As a result, the value of $y$ firstly increases with $x \in(0,0.43)$, and then decreases with $x \in(0.43,1)$. This explains the fact 
(a)

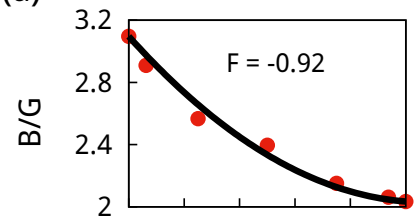

(c)

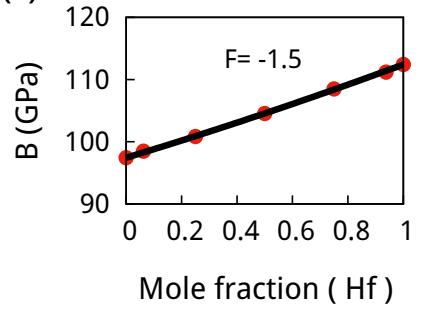

(b)

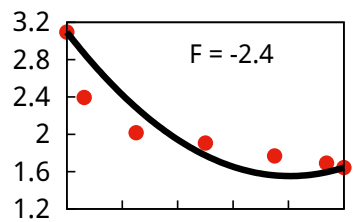

(d)

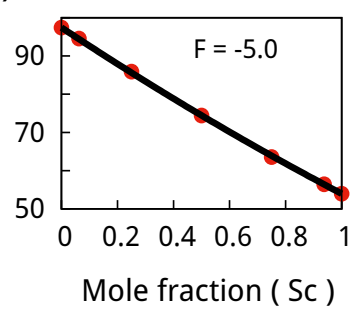

Figure 3: (Color online) Mechanical properties of Zr-Hf [(a), (c)] and Zr-Sc [(b), (d)] alloy systems. (a) and (b) are $B / G$, while (c) and (d) are bulk modulus. The red spots are calculated values and the black lines are fitting function $y=M_{Z r}(1-x)+$ $M_{X} x+x(1-x) F$.

that $y$ does not have mirror symmetry, which can be seen clearly from Fig.2(i). $B / G$ in Zr-Hf system (Fig. 3(a)) and Zr-Sc system (Fig. 3(b)) gradually decreases with concentration indicating that the ductility of Zr cannot be improved by alloying with Sc or Hf.

While some of the mechanical properties have parabolic composition dependence (Fig. 2), some other mechanical properties have linear composition dependence (Fig. 3). This behavior is mainly induced by the mechanical properties of $\mathrm{Zr}-X$ metallic bond. There is only one type of metallic bond in pure metal $\mathrm{Zr}(\mathrm{Zr}-\mathrm{Zr})$. When $X$ is introduced as an alloy element in $\mathrm{Zr}$, the second type of bond $(\mathrm{Zr}-X)$ is introduced. Normally, the mechanical properties of $\mathrm{Zr}-X$ bond are different from those of $\mathrm{Zr}-\mathrm{Zr}$ or $X-X$ bonds. Thus the mechanical properties of $\mathrm{Zr}-X$ alloys are not the superposition of pure metal $\mathrm{Zr}$ and $X$. To characterize the impact of $\operatorname{Zr}-X$ bond on mechanical properties of alloys, the relative variation of mechanical parameters $Q_{M}$ is introduced for alloy systems,

$$
Q_{M}=\frac{2 M^{Z r-X}-\left(M^{Z r-Z r}+M^{X-X}\right)}{\left(M^{Z r-Z r}+M^{X-X}\right)} 100 \%
$$

where $M^{Z r-X}$ are mechanical properties of $\mathrm{Zr}-\mathrm{X}$ bond estimated from $h c p$ unit cell with one $\mathrm{Zr}$ atom and one $X$ atom, $M^{X-X}$ are mechanical properties of $X-X$ bond estimated from $X_{2} h c p$ unit cell. Either a positive or negative of $Q_{M}$ indicates an enhanced or suppressed effect of $\operatorname{Zr}-X$ bond to the mechanical properties of $\operatorname{Zr} X$ alloys, and the numerical magnitude indicates the strength of this effect. $Q_{M}\left(M=C_{11}, C_{33}, C_{12}, C_{13}, C_{44}\right.$, $B, G, \nu$ and $B / G$ ) for $\mathrm{Zr}-X$ alloy systems are listed in Table IV, where $Q_{B}$ of $\mathrm{Zr}$-Ti system (-6.31) implies that $\mathrm{Zr}$-Ti bond has a suppressed effect on the bulk modulus of Zr-Ti system, $Q_{B}$ of Zr-Hf system (0.74) and Zr-Sc systems (0.38) show that the effect of $\mathrm{Zr}-X$ bond on bulk modulus of Zr-Hf and Zr-Hf systems is negligible. Hence bulk modulus $B$ has parabolic composition dependence in $\mathrm{Zr}$-Ti system but exhibit linear composition dependence in Zr-Hf and Zr-Sc systems, which can be observed from Fig. 2 and Fig. 3. For 
Table 4: The relative variation of mechanical parameters $\mathrm{Q}_{M}$ for $\mathrm{Zr}-X$ alloy systems, where $M$ are $C_{11}, C_{33}, C_{12}, C_{13}, C_{44}$, $B, G, \nu$ and $B / G$.

\begin{tabular}{lccccccccc}
\hline \hline & $Q_{C_{11}}$ & $Q_{C_{33}}$ & $Q_{C_{12}}$ & $Q_{C_{13}}$ & $Q_{C_{44}}$ & $Q_{B}$ & $Q_{G}$ & $Q_{\nu}$ & $Q_{B / G}$ \\
Zr-Ti system & -20.41 & -9.95 & 15.32 & -1.04 & -21.45 & -6.31 & -36.02 & 13.28 & 44.36 \\
Zr-Hf system & 0.21 & 5.05 & 6.34 & -4.51 & 3.51 & 0.74 & 2.60 & -2.02 & -7.37 \\
Zr-Sc system & 10.49 & -0.96 & -16.19 & -3.03 & 27.61 & 0.38 & 24.47 & -8.15 & -19.90 \\
\hline \hline
\end{tabular}

Zr-Ti system, the maximum and minimum of $Q_{M}$ are $Q_{B / G}(44.36)$ and $Q_{C_{13}}(-1.04)$ implying that they should have the biggest and smallest curvature, respectively, which also can be observed from Fig. 2.

Bulk modulus (Fig. 2(g), Fig. 3(c), Fig. 3(d)) as a function of alloy concentration can be perfectly described by the function (8). This phenomenon is caused by SQS templates. In fact the function (8) is supposed to describe all mechanical properties of random alloys well. However in the present work, alloys are described by SQSs that would lower the symmetry of systems and bring about fitting deviations. To confirm this idea, mechanical properties of six structures with the same composition 0.5 but different atomic arrangements for Zr-Ti were calculated where structure 1 is $h c p$ unit cell, 2-5 are SQSs-8 possessing different correlation function, 6 is SQS-16. The results (Fig. 4) indicate that bulk modulus has little to do with atomic configuration while other mechanical properties are more sensitive to atomic configuration. That is why bulk modulus as a function of alloy concentration can be better described by the function (8) than other mechanical properties. In fact, this is because bulk modulus is an average effect of atomic configuration instead of depending on a single lattice direction.

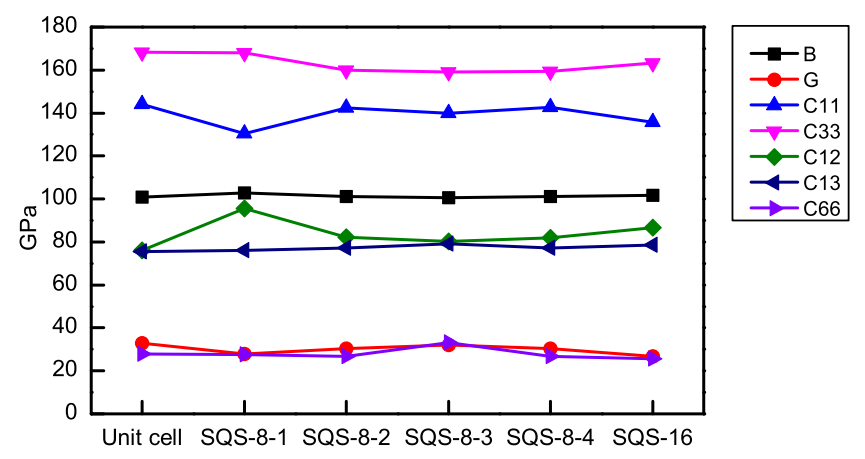



by the first-principles method gives an estimate to numerical errors in calculating elastic constants. The highest one (1.05\%) among them is regarded as global numerical errors. In Fig. 5, the $P$ for concentration 0.25, 0.5 and 0.75 are considerably higher than global numerical error (the blue line) but smaller than $8.0 \%$, which implies that the symmetry deviations bring by supercell size cutoff errors are not negligible but acceptable[11]. For Hf-Zr system, $P$ are smaller than global numerical error, which means that the symmetry deviations introduced by supercell size cutoff errors are negligible. In Fig. 5, the value of $P$ for $\mathrm{Sc}-\mathrm{Zr}$ alloy is smaller than global numerical errors except at concentrations 0.5 and 0.75 , which means in most regions of concentration symmetry deviations are negligible. Deviations at concentrations 0.25 and 0.75 are great than deviation at concentration 0.5 in $\mathrm{Zr}-\mathrm{Ti}$ and $\mathrm{Zr}$-Hf systems. One possible reason is that the correlation functions and symmetry of SQSs at 0.25 and 0.75 are lower than that of SQSs at 0.5. However this explanation is not confirmed because the trend is opposite in $\mathrm{Zr-Sc}$ system. But we notice that it consumes more time to calculate the mechanical properties at Ti concentration 0.25 (0.75) than at Ti concentration 0.5.

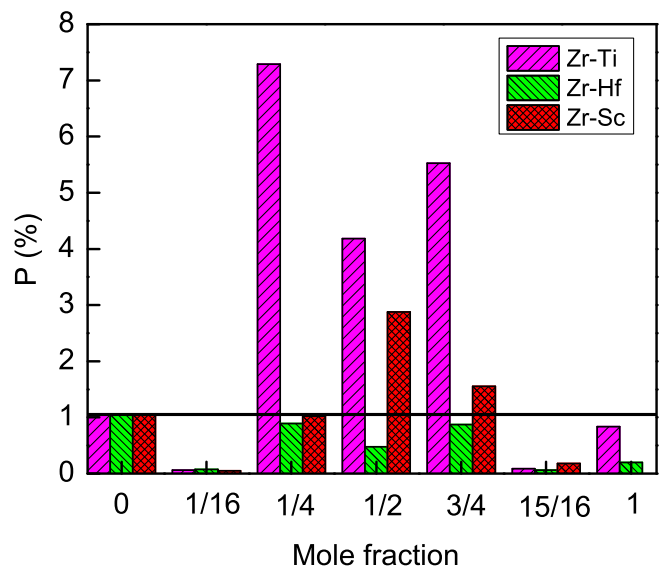

Figure 5: (Color online) Euclidean distance deviations $P=\left\|C-C^{h c p}\right\| /\|C\|$ for $\mathrm{Zr}-\mathrm{X}$ alloy systems. The black line indicates global errors of first-principles methods in calculating elastic tensor.

\section{Conclusions}

In this study, using the first-principles approach in conjunction with the special quasi-random structures (SQSs), the mechanical properties of $\operatorname{Zr}-X(X=\mathrm{Ti}$, Hf and $\mathrm{Sc}$ ) alloy systems have been systematically investigated. The dependence of the mechanical coefficient such as elastic constants and elastic modulus on alloy composition has been examined. We have found that the ductility of $\mathrm{Zr}$ can be enhanced by alloying with $\mathrm{Ti}$, whereas it would get worse if alloying with $\mathrm{Hf}$ or Sc. For these alloy systems, mechanical parameters change smoothly with alloying concentration, and can be well described by the function (8). We hope the theoretical findings presented and discussed in this work can help us to gain an in-depth understanding of the alloying effect on the mechanical properties of alloys. 


\section{Acknowledgments}

This work is supported by the National Science Foundation of China under Grant Nos. 11204310, 11534012, 11474283 and U1230202 (NSAF), the Special Funds for Major State Basic Research Project of China (973) under Grant No. 2012CB933702, One Hundred Person Project of the Chinese Academy of Sciences No. Y54N251241. The calculations were performed in Center for Computational Science of CASHIPS, the ScGrid of Supercomputing Center and Computer Net work Information Center of Chinese Academy of Science. Also this research work is supported by a Tianhe-2JK computing time award at the Beijing Computational Science Research Center (CSRC).

\section{Author Contribiutions}

Sunchao Huang, Yongsheng Zhang, Xiaoli Zhang, Zongguo Wang, Xiaoyu Yang and Zhi Zeng conceived the research as well as reviewed the manuscript. Sunchao Huang carried out the calculations and wrote the main manuscript.

[1] B. T. Wang, W. D. Li, P. Zhang, First-principles calculations of phase transition, elasticity, and thermodynamic properties for TiZr alloy, Journal of Nuclear Materials 420 (1-3) (2012) 501-507.

[2] A. C. Bagchi, G. J. Prasad, K. B. Khan, R. P. Singh, Study on Alloying Behaviour of Uranium Zirconium Alloy, Transactions of the Indian Institute of Metals 67 (3) (2013) 443-449.

[3] Y. S. Zhao, J. Z. Zhang, C. Pantea, J. Qian, L. Daemen, P. Rigg, R. Hixson, G. Gray, Y. Yang, L. Wang, Y. Wang, T. Uchida, Thermal equations of state of the $\alpha, \beta$, and $\omega$ phases of zirconium, Physical Review B 71 (18) (2005) 184119.

[4] A. Zunger, S. Wei, L. G. Ferreira, J. E. Bernard, Special Quasirandom Structure, Physical Review Letters 65 (3) (1990) 353-356.

[5] C. Jiang, C. Wolverton, J. Sofo, L.-Q. Chen, Z.-K. Liu, Physical Review B 69 (2004) 214202.

[6] A. C. Lieser, C. L. Zacherl, A. Saengdeejing, Z.-K. Liu, L. J. Kecskes, First-principles calculations and thermodynamic re-modeling of the HfW system, Calphad 38 (2012) 92-99.

[7] Q. N. Gao, J. Wang, S. L. Shang, S. H. Liu, Y. Du, Z.-K. Liu, First-principles calculations of finitetemperature thermodynamic properties of binary solid solutions in the AlCuMg system, Calphad 47 (2014) 196-210.

[8] S. Zhou, Y. Wang, L.-Q. Chen, Z.-K. Liu, R. Napolitano, Solution-based thermodynamic modeling of the NiTa and NiMoTa systems using first-principle calculations, Calphad 33 (4) (2009) 631-641.

[9] L. Zhou, D. Holec, P. H. Mayrhofer, Ab initio study of the alloying effect of transition metals on structure, stability and ductility of CrN, Journal of Physics D: Applied Physics 46 (36) (2013) 365301. 
[10] J. Von Pezold, A. Dick, M. Friák, J. Neugebauer, Generation and performance of special quasirandom structures for studying the elastic properties of random alloys: Application to Al-Ti, Physical Review B 81 (9) (2010) 094203.

[11] F. Tasnádi, M. Odén, I. A. Abrikosov, Physical Review B 85 (14) (2012) 144112.

[12] N. Wei, T. Jia, X. L. Zhang, T. Liu, Z. Zeng, X. Y. Yang, AIP Advances 4 (2014) 057103.

[13] S. C. Huang, X. L. Zhang, Y. S. Zhang, S. J. Hou, X. Y. Yang, Z. Zhi, Investigations of the mechanical properties of the Zr8Ti8 random alloy, Int. J. Mod. Phys. C 27 (7) (2016) 1650076.

[14] V. Y. Trubitsin, E. B. Dolgusheva, Calculation of the P-T phase diagram and tendency toward decomposition in equiatomic TiZr alloy., Journal of Physics: Condensed Matter 22 (46) (2010) 465401.

[15] G. Kresse, J. Furthmüller, Efficiency of ab-initio total energy calculations for metals and semiconductors using a plane-wave basis set, Computational Materials Science 6 (1996) 15-50.

[16] J. P. Perdew, K. Burke, M. Ernzerhof, Generalized Gradient Approximation Made Simple, Physical Review Letters 77 (3) (1996) 3865-3868.

[17] Y. Le Page, P. Saxe, Symmetry-general least-squares extraction of elastic data for strained materials from ab initio calculations of stress, Physical Review B 65 (10) (2002) 104104.

[18] D. Shin, R. Arróyave, Z.-K. Liu, A. Van De Walle, Thermodynamic properties of binary hcp solution phases from special quasirandom structures, Physical Review B 74 (2) (2006) 024204.

[19] S. Wei, L. G. Ferreira, J. E. Bernard, A. Zunger, Electronic properties of random alloys: Special quasirandom structures, Physical Review B 42 (15).

[20] M. Moakher, A. N. Norris, The Closest Elastic Tensor of Arbitrary Symmetry to an Elasticity Tensor of Lower Symmetry, Journal of Elasticity 85 (3) (2006) 215-263.

[21] G. Ghosh, A. V. D. Walle, M. Asta, First-principles calculations of the structural and thermodynamic properties of bcc, fcc and hcp solid solutions in the Al-TM ( $\mathrm{TM}=\mathrm{Ti}, \mathrm{Zr}$ and Hf ) systems : A comparison of cluster expansion and supercell methods, Acta Materialia 56 (2008) 3202-3221.

[22] C. Wolverton, Crystal structure and stability of complex precipitate phases in al-cu-mg-(si) and al-znmg alloys, Acta Materialia 49 (16) (2001) 3129-3142.

[23] S. Shang, A. Saengdeejing, Z. Mei, D. Kim, H. Zhang, S. Ganeshan, Y. Wang, Z. Liu, First-principles calculations of pure elements: Equations of state and elastic stiffness constants, Computational Materials Science 48 (4) (2010) 813-826.

[24] W. M. Haynes, D. R. Lide, CSC Handbook of Chemistry and Physics (CRC Press, Boca Raton) 92nd (2011) 12-35. 
[25] H.-C. Hsu, S.-C. Wu, Y.-C. Sung, The structure and mechanical properties of as-cast Zr-Ti alloys, Journal of Alloys and Compounds 488 (1) (2009) 279-283. 

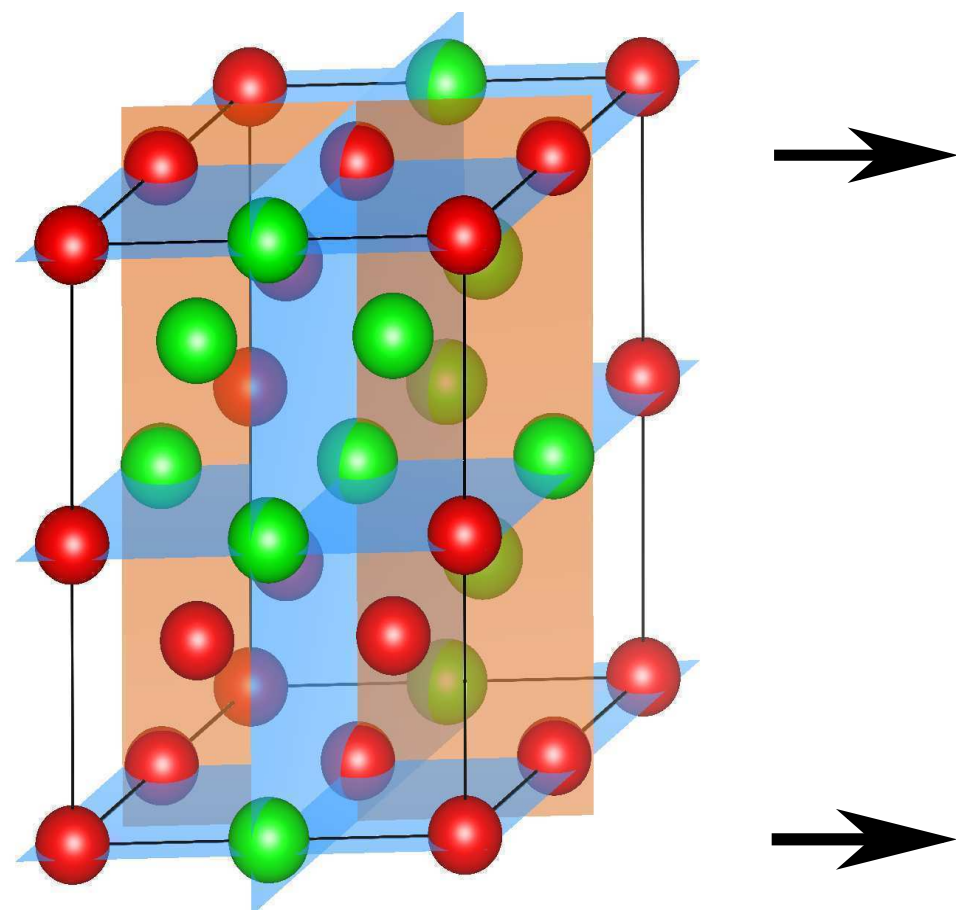
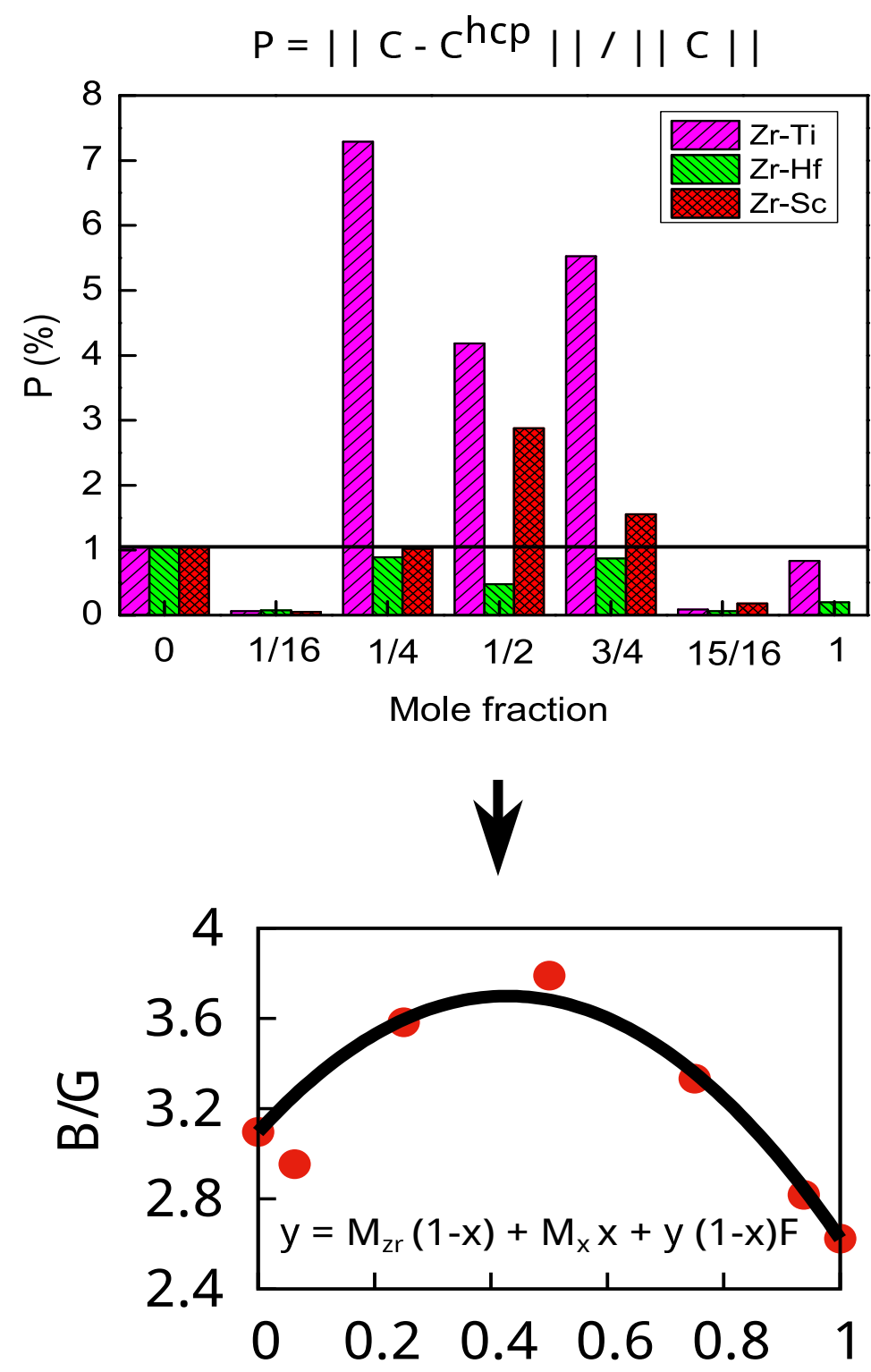

Mole Fraction of (Ti)

Special quasi-random structure (SQS) 